\title{
H-alpha monitoring of OJ 287 in 2005-08 ${ }^{\star}$
}

\author{
K. Nilsson ${ }^{1,2}$, L. O. Takalo ${ }^{1}$, H. J. Lehto ${ }^{1}$, and A. Sillanpää ${ }^{1}$ \\ 1 Tuorla Observatory, Department of Physics and Astronomy, University of Turku, Väisäläntie 20, 21500 Piikkiö, Finland \\ e-mail: kani@utu.fi \\ 2 Finnish Centre for Astronomy with ESO (FINCA), University of Turku, Väisäläntie 20, 21500 Piikkiö, Finland \\ Received 4 February 2010 / Accepted 9 April 2010
}

\section{ABSTRACT}

\begin{abstract}
We present the results of $\mathrm{H} \alpha$ monitoring of the BL Lac object OJ 287 with the VLT during seven epochs in 2005-08. We were able to detect five previously undetected narrow emission lines, $\lambda \lambda 6548,6583[\mathrm{NII}], \lambda 6563 \mathrm{H} \alpha$ and $\lambda \lambda 6716,6731$ [SII] during at least one of the epochs and a broad $\mathrm{H} \alpha$ feature during two epochs. The broad $\mathrm{H} \alpha$ luminosity was a factor $~ 10$ lower in 2005-08 than in 1984 when the line was previously detected and a factor $\sim 10$ lower than what is observed in quasars and Seyfert galaxies at the same redshift. The data are consistent with no change in the position or luminostity of the $\mathrm{H} \alpha$ line in 2005-08. The width of the $\mathrm{H} \alpha$ line was $4200 \pm 500 \mathrm{~km} \mathrm{~s}^{-1}$, consistent with the width in 1984 .
\end{abstract}

Key words. galaxies: active - BL Lacertae object: individual: OJ 287 - galaxies: nuclei

\section{Introduction}

The BL Lac object OJ $287(z=0.306)$ has received some attention after the discovery of the recurring outbursts every $\sim 12$ years in the optical data over the past century (Sillanpää et al. 1988). This observation has led many authors to consider a binary black hole model for OJ 287 (Sillanpää et al. 1988; Lehto \& Valtonen 1996; Katz 1997; Sundelius et al. 1997; Villata et al. 1998; Valtaoja et al. 2000; Valtonen et al. 2006; Valtonen 2007), but see also Villforth et al. (2010). The exact mechanism of the outburst differs from one model to another. Some models attribute the flux increase to changes of the direction of the relativistic jet (e.g. Katz 1997), other models to the impact of the secondary black hole onto the accretion disk of the primary followed by tidal effects and increased luminosity of the jet (e.g. Lehto \& Valtonen 1996). The most detailed model so far (Valtonen 2007) suggests a very high mass for the primary black hole $M=1.8 \times 10^{10} M_{\odot}$ and a very eccentric orbit for the secondary $(e=0.66)$. To explain the aperiodicity of the outbursts the precession of the orbit has to be very strong, $\Delta \phi=40^{\circ}$ per orbit, in this model.

Testing these models was so far concentrated mainly on the timing of the outbursts with broadband photometric observations (e.g. Valtonen et al. 2009) with other aspects receiving less attention. One interesting constraint to the models could be obtained by monitoring the emission line variability during the outbursts. The luminosity of the emission lines could change in response to varying continuum radiation or, if the size of the secondary black hole orbit and the characteristic broad line region (BLR) size are of the same order, the secondary black hole could affect the velocity field of the BLR. Furthermore, if the BLR velocity dispersion was measured, it could be used to constrain the mass of the primary black hole in OJ 287 . However, there are

^ Based on observations collected at the European Organization for Astronomical Research in the Southern Hemisphere, Chile, proposals 075.B-0527, 076.B-0028, 077.B-0015, 078.B-0030, 079.B-0014, 380.B-0665 and 381.B-0050. no published results of spectroscopic monitoring of OJ 287 in the literature. Thus we started a project in 2005 at the VLT to monitor the broad line region of OJ 287 during the expected outbursts in 2005-08 when the two black holes were expected to be at closest approach by various models. The main aim of this project was to see if any changes could be observed in the BLR of OJ 287 during the expected closest approach of the binary.

This kind of monitoring project is quite challenging because the emission lines are very weak in BL Lacs by definition. There are only two reported detections of broad lines in OJ 287 in the literature. Stickel et al. (1989) detected the $\mathrm{H} \beta$ line with a $F W H M$ of $56 \AA$ with an equivalent width $(E W)$ of $1.1 \AA$. Sitko \& Junkkarinen (1985) also detected a weak $\mathrm{H} \beta$, but the most notable feature in their spectrum is the prominent broad $(F W H M \sim 120 \AA) \mathrm{H} \alpha$ line. It is impossible to know if these detections represent typical levels or very high states of the lines. Given the weakness of emission lines in BL Lacs in general, the latter alternative seems more probable as non-detections are not always reported. Based on this past information the $\mathrm{H} \alpha$ line was chosen for the monitoring because it can be easily detected with modern spectrographs at sufficiently large telescopes if the luminosity of the line is close to what was observed by Sitko \& Junkkarinen (1985). The results of this monitoring are reported in this paper.

Throughout this paper we use the cosmology $H_{0}=$ $70 \mathrm{~km} \mathrm{~s}^{-1} \mathrm{Mpc}^{-1}, \Omega_{\mathrm{M}}=0.3$ and $\Omega_{\Lambda}=0.7$.

\section{Observations and data reduction}

The observations were made with the FORS2 instrument (Appenzeller et al. 1998) attached to the VLT-UT1 (Antu) in service mode during seven epochs in 2005-08. Throughout the whole period the same instrumental setup was used, employing the $1028 \mathrm{z}$ grism with the OG590 order separation filter. The wavelength range covered by the $1028 \mathrm{z}$ grism is $\lambda \lambda 7730$ $9480 \AA$ and the resolution with the 2 "slit was $8.1 \AA$. The observations are summarized in Table 1 . The exposure time in Table 1 
Table 1. Summary of the observations. The last column gives the signalto-noise at $8700 \AA$.

\begin{tabular}{llllll}
\hline \hline Epoch & Date & $\begin{array}{l}t_{\exp } \\
(\mathrm{s})\end{array}$ & $\begin{array}{l}F W H M \\
(\operatorname{arcsec})\end{array}$ & Airmass & $S / N$ \\
\hline 1 & $2005-04-05$ & 1200 & 0.9 & 1.46 & 280 \\
2 & $2005-11-19$ & 1800 & 0.9 & 1.61 & 330 \\
3 & $2006-04-02$ & 1500 & 0.6 & 1.41 & 400 \\
4 & $2006-12-06$ & 1800 & 0.9 & 1.50 & 315 \\
5 & $2007-04-04$ & 2500 & 0.7 & 1.43 & 440 \\
6 & $2008-01-05$ & 2500 & 0.8 & 1.41 & 560 \\
7 & $2008-04-08$ & 2640 & 1.1 & 1.42 & 410 \\
\hline
\end{tabular}

gives the total exposure time of 2-5 exposures of OJ 287 made a each epoch.

The reduction of the spectra was made with IRAF. First a bias frame was subtracted from the two-dimensional CCD spectra after which they were divided by a flat field frame derived from continuum lamp exposures. A two-dimensional wavelength calibration solution was derived from strips of data separated by 5 arcsec in the spatial direction using the same 14 night-sky emission lines in each strip. This solution was used to rectify the image before subtracting the night-sky emission lines. After the night-sky subtraction the one-dimensional spectrum of OJ 287 was extracted by tracing the spectrum of OJ 287 on the CCD and integrating the light within a 7.5 arcsec aperture. Optimal extraction was used at this phase to obtain maximum signal-to-noise.

The spectra were calibrated using five spectrophotometric calibration stars (LTT 4816, EG 274, LTT 7379, LTT 6248 and GD 108) obtained with the same instrumental setup as here during the observations or from the ESO archive observations made in 2005-06. The average sensitivity curve was derived form these five stars and applied to the spectra of OJ 287. An extinction correction was made to each spectrum using a standard atmospheric extinction curve and the airmass at the time of the observation. Some of the nights were affected by thin clouds, whose absorption cannot be accounted for by the above calibration procedure.

The calibrated spectra exhibit absorption bands from atmospheric water vapor, which are particularly prominent at $\lambda \lambda 8100-8400 \AA$ and $\lambda \lambda 8800-9300 \AA$ and whose strength varied from one epoch to another. To correct for the atmospheric absorption bands a template was derived from the spectrum of the nearby star 10 (Fiorucci \& Tosti 1996) observed simultaneously with OJ 287 on 2008-01-05. This template was scaled appropriately to obtain the best correction for each epoch. During this phase it became evident that due to the variable nature of the atmospheric absorption bands they cannot be completely removed at their cores. The wavelength regions most affected by this effect were excluded from further analysis (see below).

\section{Analysis and results}

Figure 1 shows an example of a calibrated spectrum of OJ 287 obtained during the monitoring campaign. The spectrum shows two noisy areas at $\lambda \lambda 8100-8400 \AA$ and $\lambda \lambda 8850-9200 \AA$ due to the imperfect subtraction of the atmospheric absorption bands. The spectra also show two areas populated by weak emission lines: at $\lambda \sim 8570 \AA$ is the $\lambda \lambda 6548,6583$ [NII] $+\lambda 6563 \mathrm{H} \alpha$ group and at $\lambda \sim 8780 \AA$ the $\lambda \lambda 6716,6731$ [SII] lines. Horizontal bars in Fig. 1 indicate the wavelength areas representing "pure" continuum. These areas, $7750-8100 \AA$, 8400-8460 ̊, 8680-8750 ̊

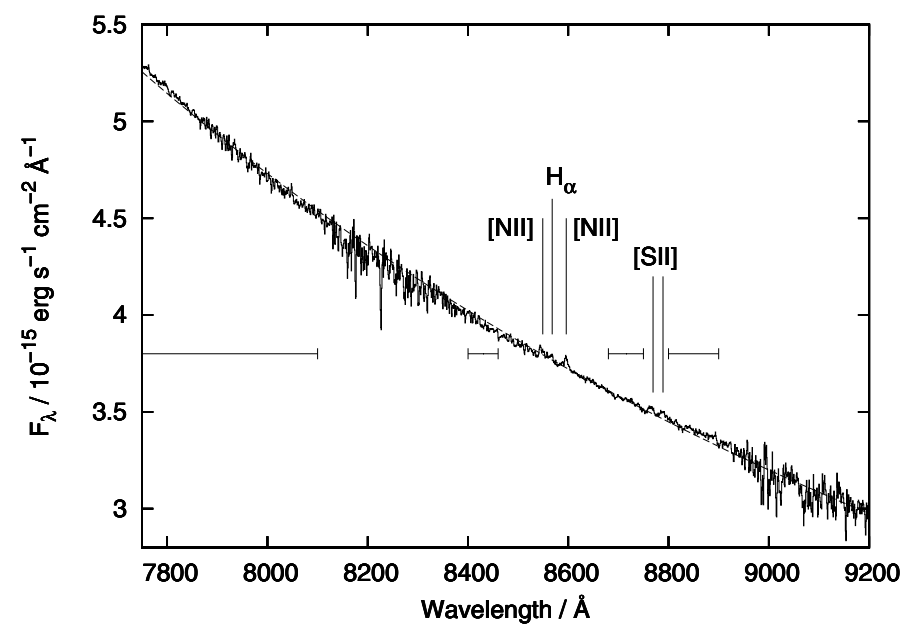

Fig. 1. Spectrum of OJ 287 on 2008-01-05. The dashed line is a powerlaw fit to pure continuum parts of the spectrum, indicated by horizontal bars. The spectral lines identifiable in the spectrum have also been marked.

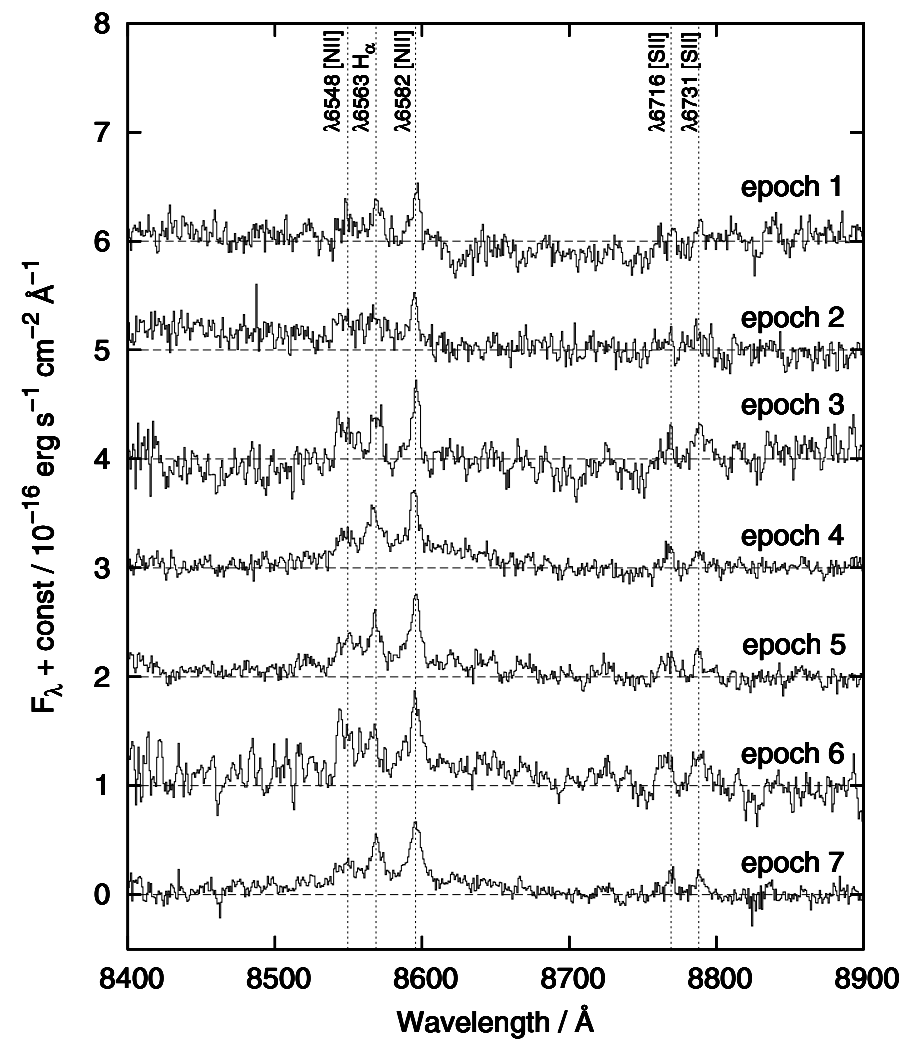

Fig. 2. Continuum-subtracted spectra of OJ 287 during the monitoring. Individual spectra have been shifted by $10^{-16} \mathrm{erg} \mathrm{s}^{-1} \mathrm{~cm}^{-2} \AA^{-1}$ in vertical direction for clarity.

and 8800-8900 $\AA$ are used in all subsequent work for continuum fitting.

The overall shape of the continuum follows a power-law very well as indicated by the power-law fit in Fig. 1. In all seven spectra the maximum deviation from the power-law fit is $1 \%$. However, because the main interest of this work is on the line variations of OJ 287, a second order polynomial was chosen for the continuum fit as it gives about two times smaller residuals than the power-law fit and still retains the smooth and monotonous shape of the power-law spectrum. Figure 2 shows 
Table 2. Results of the search for significant spectral features.

\begin{tabular}{|c|c|c|c|c|c|c|c|c|c|}
\hline \multirow{4}{*}{ Epoch } & \multirow{4}{*}{ JD - 2450000} & \multirow{4}{*}{$\begin{array}{l}E W \text { limit } \\
(\AA) \\
(\AA \text { redshifted })\end{array}$} & \multicolumn{5}{|c|}{ Detected? } & \multirow{4}{*}{$\begin{array}{l}\text { Broad } \\
\text { features }\end{array}$} & \multirow{4}{*}{$\begin{array}{l}\text { Other } \\
\text { features }\end{array}$} \\
\hline & & & {$[\mathrm{NII}]$} & $\mathrm{H} \alpha$ & {$[\mathrm{NII}]$} & {$[\mathrm{SII}]$} & [SII] & & \\
\hline & & & 6548 & 6563 & 6583 & 6716 & 6731 & & \\
\hline & & & 8550 & 8569 & 8595 & 8769 & 8789 & & \\
\hline 1 & 3466.56759 & 0.082 & no & yes & yes & no & no & $\ldots$ & absorption at $8738 \AA$ \\
\hline 2 & 3693.83049 & 0.092 & no & no & yes & no & no & $\ldots$ & $\ldots$ \\
\hline 3 & 3827.53441 & 0.072 & no & no & yes & no & no & $\ldots$ & $\ldots$ \\
\hline 4 & 4075.80737 & 0.059 & yes & yes & yes & yes & yes & emission at $8538-8642 \AA$ & $\ldots$ \\
\hline 5 & 4194.55954 & 0.051 & yes & yes & yes & no & yes & emission at $8546-8586 \AA$ & absorption at $8058 \AA$ \\
\hline 6 & 4470.76465 & 0.067 & yes & yes & yes & no & no & emission at $8546-8570 \AA$ & $\ldots$ \\
\hline 7 & 4564.53963 & 0.061 & yes & yes & yes & no & no & emission at $8538-8602 \AA$ & $\ldots$ \\
\hline
\end{tabular}

the continuum-subtracted spectra during the seven epochs of the monitoring. We note that the continuum level varied by a factor of 2.6 during the monitoring.

During at least two of the epochs (four and seven) a broad spectral feature is seen at the location of the $\mathrm{H} \alpha$-[NII] lines and epochs five and six show a hint of a similar feature. However, as is evident from Fig. 2, the continuum level is fluctuating around the mean level due to calibration errors etc. Because these fluctuations could be wrongly interpreted as true features, it is important to study the statistical properties of the fluctuations more closely.

To study the significance of the spectral features in Fig. 2 we employed the method by Sbarufatti et al. (2005). This method consists of dividing the spectrum into spectral bins, computing the equivalent width $(E W)$ of each bin and deriving the rms fluctuations of the $E W$ through the spectrum. Any $E W$ exceeding a pre-defined rms limit (e.g. $3 \sigma$ ) is taken as a sign of significant emission or absorption line warranting further study. Sbarufatti et al. (2005) computed the continuum level for each bin from two adjacent bins, which works fine for narrow lines, but very broad features may be missed. Thus the method by Sbarufatti et al. (2005) was slightly modified by using the fitted continuum value instead of the adjacent bins as the continuum estimate. The rms noise in the $E W$ was computed from the pure continuum areas marked with horizontal bars in Fig. 1. A bin size of $8 \AA$ was used in the computations and as in Sbarufatti et al. (2005) any $E W$ value thrice the rms $E W$ was interpreted as a significant spectral feature. The two areas where the atmospheric line correction produces too much noise (Fig. 1) were excluded from the analysis.

The results of this analysis are shown in Table 2. Column 3 of the table gives the derived $3 \sigma E W$ limit used to detect significant features. Columns 3-7 list whether the five narrow emission lines seen in the spectrum were formally detected or not. The last two columns give wavelength regions where broad spectral features, defined as at least three adjacent bins with significant emission, were detected and the last column gives any other significant spectral features detected.

Of the five visually identified narrow emission lines only $\lambda 6583$ [NII] is consistently detected throughout the campaign. The rest are detected more sporadically, especially the [SII] lines are mostly below the $3 \sigma$ level. There is a clear indication of broad emission on top of the $[\mathrm{NII}] / \mathrm{H} \alpha$ lines, which is most naturally explained as broad $\mathrm{H} \alpha$ emission. This broad emission component is particularly clear at epoch four. In addition to the lines in Table 2 the only detected significant features are two weak $(3 \sigma)$ absorption features at 8058 and $8738 \AA$. These lines do not correspond to any commonly observed spectral lines at the redshift of OJ 287.

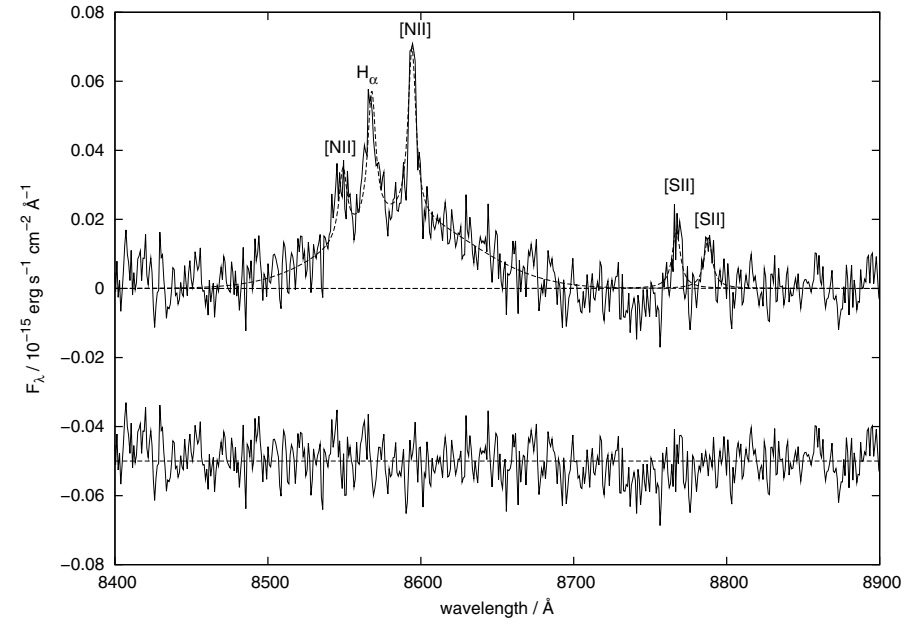

Fig. 3. Example of the line fitting (epoch four). The data are shown as a continuous line and the model fit as a dashed line. The lower part shows the residuals of the fit, shifted downwards by $0.05 \times 10^{-15}$.

To study the properties and significance of the emission lines further, model fits were made to the observed spectra. The narrow lines were modeled by a Lorentzian profile and the broad $\mathrm{H} \alpha$ line with a Gaussian profile. Only significant features were fitted and the broad $\mathrm{H} \alpha$ was fitted simultaneously with the narrow lines on top of it. Figure 3 shows the result of the line fitting on epoch four. Table 3 lists the values of the fitted parameters and their associated errors.

The error bars were estimated by performing model fits to 500 simulated spectra for each epoch. The simulated data sets contained all significant emission features for that particular epoch. The noise in the spectra was simulated by performing a Fourier transform to the continuum-subtracted pure continuum parts of the observed spectra, randomizing the phases and transforming back to obtain the simulated noise. In this way the statistical properties of the noise were preserved and the fluctuations the simulated spectra resembled closely the observed fluctuations. Examining the distributions of the simulation results it was found that for some parameters (e.g. the FWHM of the narrow lines) the distribution was not symmetrical, but skewed towards higher values. For this reason asymmetric error bars are given in Table 3. The quoted error range includes $67 \%$ of the simulated values, computed separately above and below the median value. The broad $\mathrm{H} \alpha$ was considered significant only if the flux of the line could be determined to better than $3 \sigma$ accuracy, where $\sigma$ is the lower error bar. Table 3 lists only significant detections of the broad $\mathrm{H} \alpha$ (epochs four and seven). We also computed the $3 \sigma$ upper limits for the broad $\mathrm{H} \alpha$ line by creating simulated data 
Table 3. Results of the line fitting.

\begin{tabular}{|c|c|c|c|c|}
\hline Epoch & line & $\begin{array}{c}F W H M \\
(\AA)\end{array}$ & $\begin{array}{c}\lambda_{c} \\
(\AA)\end{array}$ & $\begin{array}{c}\text { Flux } \\
\left(10^{-16} \mathrm{erg}\right. \\
\left.\mathrm{s}^{-1} \mathrm{~cm}^{-2}\right)\end{array}$ \\
\hline \multirow[t]{2}{*}{1} & $\mathrm{H} \alpha$ narrow & $6.1_{-20}^{+3.1}$ & $8569.3_{-06}^{+1.0}$ & $4.0^{+2.0}$ \\
\hline & 6583 [NII] & & $8596.1_{-1.1}^{-0.8}$ & $4.4_{-1.7}^{+2.6}$ \\
\hline 2 & 6583 [NII] & $7.7_{-3.3}^{+7.2}$ & $8594.6_{-0.9}^{+1.1}$ & $5.8_{-3.2}^{+5.8}$ \\
\hline 3 & 6583 [NII] & $4.7_{-1.7}^{+2.7}$ & $8595.5_{-0.7}^{+1.1}$ & $5.3_{-2.0}^{+2.9}$ \\
\hline \multirow[t]{6}{*}{4} & 6548 [NII] & $6.4_{-1.2}^{+1.4}$ & $8549.1_{-1.6}^{+2.0}$ & $2.1_{-0.7}^{+0.9}$ \\
\hline & $\mathrm{H} \alpha$ narrow & & $8567.9_{-1.1}^{+1.1}$ & $3.8_{-1.0}^{+1.0}$ \\
\hline & $\mathrm{H} \alpha$ broad & $106_{-14}^{+22}$ & $8589.7_{-12.4}^{+15.1}$ & $22.5_{-4.1}^{+3.0}$ \\
\hline & 6583 [NII] & & $8594.2_{-0.9}^{+0.9}$ & $4.9_{-1.1}^{+1.3}$ \\
\hline & 6716 [SII] & & $8767.0_{-1.4}^{+0.9}$ & $1.7_{-0.6}^{+0.1}$ \\
\hline & 6731 [SII] & & $8787.9_{-1.9}^{+1.3}$ & $1.5_{-0.5}^{+0.0}$ \\
\hline \multirow[t]{4}{*}{5} & 6548 [NII] & $7.4_{-1.6}^{+2.0}$ & $8549.8_{-1.6}^{+2.0}$ & $3.4_{-1.3}^{+1.8}$ \\
\hline & $\mathrm{H} \alpha$ narrow & & $8567.5_{-1.4}^{+1.7}$ & $4.5_{-1.6}^{+1.8}$ \\
\hline & 6583 [NII] & & $8594.2_{-1.2}^{+1.4}$ & $6.7_{-1.8}^{+1.0}$ \\
\hline & 6731 [SII] & & $8788.5_{-2.2}^{+1.8}$ & $2.4_{-0.9}^{+1.0}$ \\
\hline \multirow[t]{3}{*}{6} & 6548 [NII] & $8.9_{-3.0}^{+9.9}$ & $8547.1_{-3.2}^{+9.0}$ & $5.7_{-2.9}^{+4.0}$ \\
\hline & $\mathrm{H} \alpha$ narrow & & $8564.7_{-8.4}^{+5.2}$ & $4.3_{-3.7}^{+3.9}$ \\
\hline & 6583 [NII] & & $8595.4_{-1.8}^{+1.5}$ & $8.9_{-3.0}^{+7.0}$ \\
\hline \multirow[t]{4}{*}{7} & 6548 [NII] & $6.9_{-1.8}^{+2.5}$ & $8550.1_{-3.0}^{+3.9}$ & $1.7_{-1.0}^{+1.0}$ \\
\hline & $\mathrm{H} \alpha$ narrow & & $8568.5_{-1.5}^{+2.2}$ & $3.8_{-1.0}^{+1.8}$ \\
\hline & $\mathrm{H} \alpha$ broad & $144_{-19}^{+26}$ & $8573.8_{-14.2}^{+13.1}$ & $23.5_{-3.8}^{+4.0}$ \\
\hline & 6583 [NII] & & $8595.6_{-1.0}^{+1.0}$ & $6.0_{-1.4}^{+2.1}$ \\
\hline
\end{tabular}

sets of $\sim 300$ simulations, each containing increasing contribution of the broad $\mathrm{H} \alpha$ and determining the line flux which could be detected with $3 \sigma$ significance.

The rms scatter in the position of the $\lambda 6583$ [NII] line is $0.8 \AA$, consistent with the $\sim 1 \AA$ error bars derived by the error simulations. The average redshift derived from the $\lambda 6583$ [NII] line is $0.3056 \pm 0.0001$, consistent with the earlier redshift $z=0.306$ (Miller et al. 1978; Sitko \& Junkkarinen 1985). The error in the redshift represents the internal precision of the measurements without systematic effects, which are expected to be small because the wavelength calibration was derived from sky lines exposed simultaneously with the OJ 287 spectrum.

Figure 4 shows the position and flux density of the $\lambda 6583$ [NII] and $\mathrm{H} \alpha$ lines. The $\lambda 6583$ [NII] line is not expected to show any variations over the timescales considered here, which is also confirmed by our results: no variability larger than the error bars is observed. However, the error bars of the flux densities are quite large. The broad $H_{\alpha}$ line shows no significant changes in position or flux between the two epochs it was detected. As the upper limits show, the data are consistent with no change in the broad $\mathrm{H} \alpha$ line flux throughout the whole monitoring campaign. Thus we find no evidence of changes in the BLR in our data. However, there is a big difference to the line flux observed by Sitko \& Junkkarinen (1985), who reported a $\mathrm{H} \alpha$ line flux of $2.4 \times 10^{-14} \mathrm{erg} \mathrm{cm}^{-2} \mathrm{~s}^{-1}$, which is a factor of $\sim 10$ higher than in 2005-08. The difference remains even after subtracting the summed contribution of the [NII] and narrow $\mathrm{H} \alpha$ lines, $12 \times 10^{-16} \mathrm{erg} \mathrm{cm}^{-2} \mathrm{~s}^{-1}$, which were unresolved by Sitko \& Junkkarinen (1985).

The weighted average of the broad $\mathrm{H} \alpha$ line width is $120 \pm$ $14 \AA$, which corresponds to $4200 \pm 500 \mathrm{~km} / \mathrm{s}$ at the redshift of OJ 287. Sitko \& Junkkarinen (1985) do not give the line width, but estimating from their Fig. 2 it is $\sim 120 \AA$, i.e. the same as observed here.
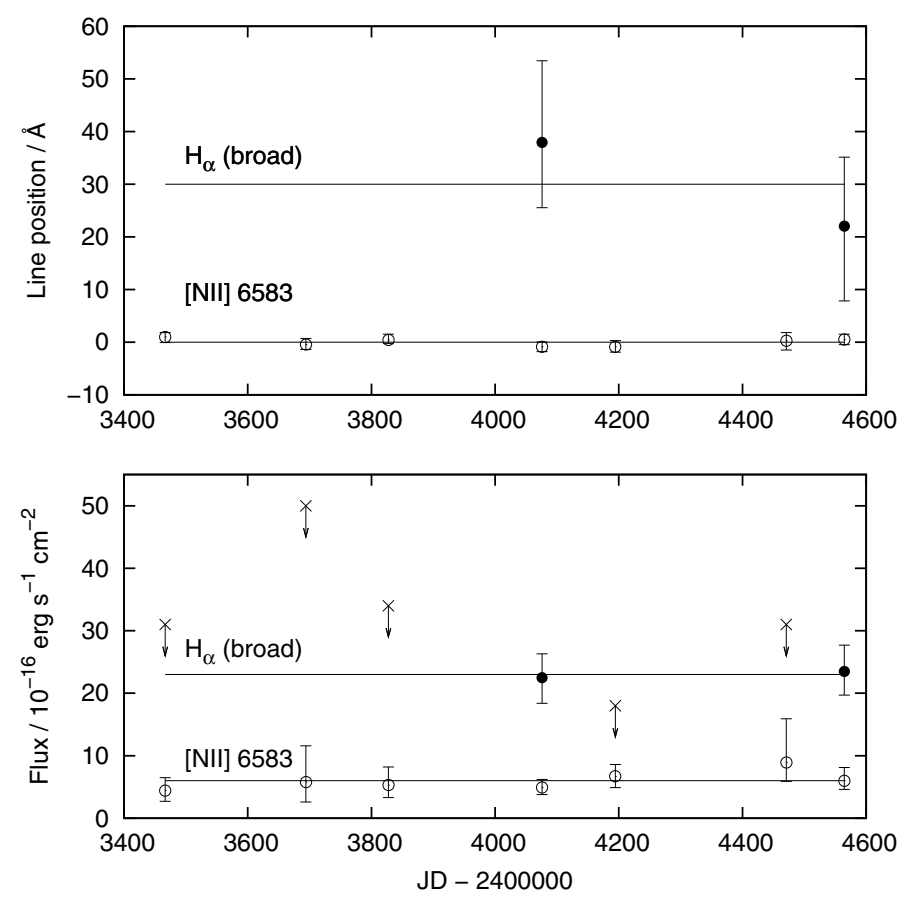

Fig. 4. Line positions (upper panel) and flux density (lower panel) of the broad $\mathrm{H} \alpha$ and $\lambda 6583$ [NII] lines. In both panels the results for the $\mathrm{H} \alpha$ line are shown with closed symbols (upper limits with crosses) and the $\lambda 6583$ [NII] line with open symbols. Horizontal lines represent average values. In the upper panel the the average value has been subtracted and the plot for the $\mathrm{H} \alpha$ moved $30 \AA$ up for clarity.

\section{Discussion}

The most notable result of our monitoring is the dramatic decrease in broad $\mathrm{H} \alpha$ luminosity between 1984 and 2005-08. In Fig. 5 we show the continuum and broad $\mathrm{H} \alpha$ line flux of OJ 287 in 1980-2010. In the upper panel three outburst seasons of OJ 287 can be seen: 1982-84, 1994-95 and 2005-08. The high $\mathrm{H} \alpha$ flux appeared just after the strong outbursts in 198283, 290 days after the second peak. Unfortunately, the sampling is too poor to draw any conclusions on the possible connection between continuum and BLR luminosity in OJ 287. Such a connection would not even be necessarily expected because in BL Lac objects the vast majority of the continuum arises from the jet and is highly beamed by relativistic effects due to the small angle between the line of sight and the jet axis. Thus the observed continuum variations are not necessarily connected to the changes of the continuum source illuminating the $\mathrm{H} \alpha$ emitting clouds, most likely the inner parts of the accretion disk. It is nevertheless an intriguing observation that the high line luminosity in 1984 was observed right after a luminous continuum outburst. Given the weakness of the broad $\mathrm{H} \alpha$ line we are unable to study a possible connection between BLR velocity field and the suggested periastron of the secondary black hole in 2005-07 (Valtonen 2007).

The average line luminosities in 2005-08 were $\log \left(L / \mathrm{erg} \mathrm{s}^{-1}\right)=41.8,41.1,41.2$ and 40.8 for broad $\mathrm{H} \alpha$, narrow $\mathrm{H} \alpha, \lambda 6583[\mathrm{NII}]$ and $\lambda 6548[\mathrm{NII}]$, respectively. The $\mathrm{H} \alpha$ luminosities of Seyfert galaxies and quasars are generally much higher as shown by he comparisons in Table 4 and Fig. 6. The quasar data were obtained from Schneider et al. (2007), who give data of 77429 quasars from the Fifth Data Release of the Sloan Digital Sky Survey (Adelman-McCarthy et al. 2007). We selected quasars at the redshift interval $z=0.30-0.31$ (147 quasars) and obtained the $\mathrm{H} \alpha$ line fluxes and widths from 

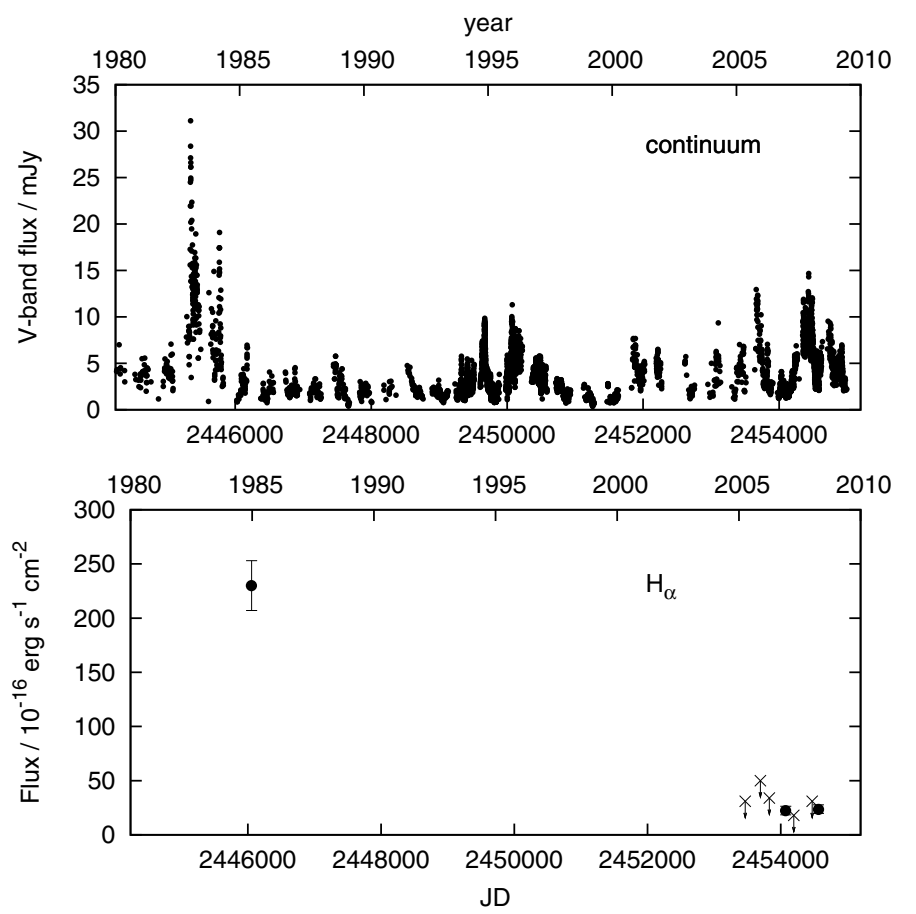

Fig. 5. Continuum (upper panel) and $\mathrm{H} \alpha$ (lower panel) flux of OJ 287 in 1980-2010. In the lower panel crosses with arrows mark $3 \sigma$ upper limits and solid circles detections. The error bar of the leftmost point in the lower panel has been arbitrarily set to $10 \%$.

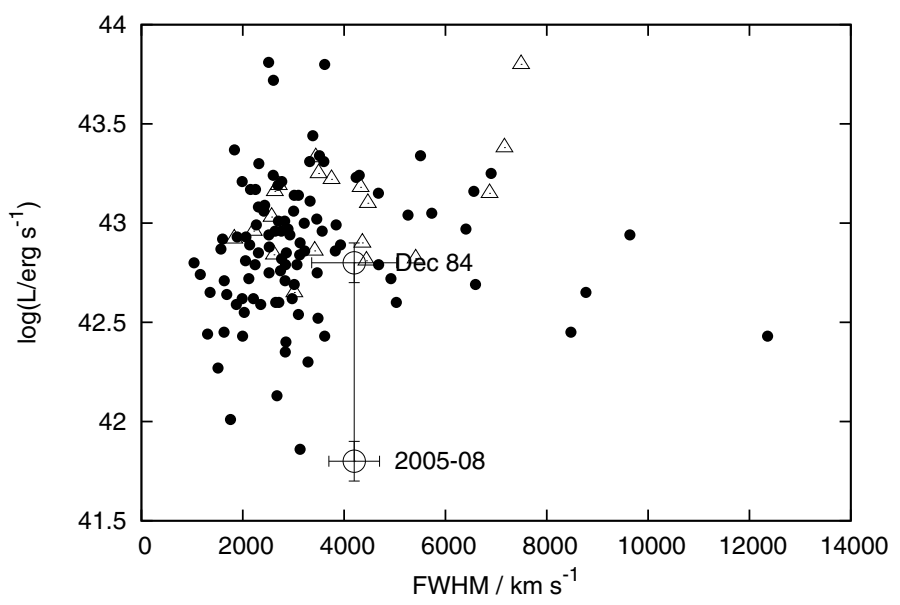

Fig. 6. Comparison of broad $\mathrm{H} \alpha$ luminosities of OJ 287 (open circles connected by a line), $z=0.30-0.31$ quasars from Schneider et al. (2007, filled circles) and $z=0.36$ Seyfert galaxies from McGill et al. (2008, open triangles).

the SpecLine tables in the SDSS archive. Figure 6 shows only quasars with $F W H M>1000 \mathrm{~km} \mathrm{~s}^{-1}$ (101 quasars). In addition we plot data for $17 z=0.36$ Seyfert galaxies in McGill et al. (2008). As can be seen from Fig. 6 the H $\alpha$ luminosity of OJ 287 was lower than in typical quasars and Seyfert galaxies by a factor of $\sim 10$ in 2005-08. In December 1984, however, the $\mathrm{H} \alpha$ luminosity was comparable to that of quasars and Seyferts. We finally note that the luminosities of the two narrow lines $\lambda 6548$
Table 4. Comparison of line luminosities of OJ 287 to those of quasars and Seyfert galaxies.

\begin{tabular}{llll}
\hline \hline & \multicolumn{3}{c}{$\log L$} \\
Line & OJ 287 & Quasars & Seyferts \\
\hline $\mathrm{H} \alpha$ broad & 41.8 & $42.9 \pm 0.4$ & $43.1 \pm 0.3$ \\
$\lambda 6548[\mathrm{NII}]$ & 40.8 & $41.3 \pm 0.8$ & $\ldots$ \\
$\lambda 6583[\mathrm{NII}]$ & 41.2 & $41.4 \pm 0.7$ & $\ldots$ \\
\hline
\end{tabular}

and $\lambda 6583$ [NII], however, are comparable to the narrow-line luminosities of the quasars in Schneider et al. (2007).

\section{Summary}

We have presented high S/N spectra of the BL Lac object OJ 287 during seven epochs in 2005-08. Our results can be summarized as follows:

1) We were able to detect five narrow emission lines, $\lambda \lambda 6548,6583[\mathrm{NII}], \lambda 6563 \mathrm{H} \alpha$ and $\lambda \lambda 6716,6731$ [SII] during at least one of the epochs and a broad $\mathrm{H} \alpha$ feature during two epochs. The luminosities of the [NII] lines are comparable to those in quasars at the same redshift, whereas the broad $\mathrm{H} \alpha$ line is a factor of $\sim 10$ less luminous than the $\mathrm{H} \alpha$ line in quasars and Seyfert galaxies.

2) The luminosity of the broad $\mathrm{H} \alpha$ line was a factor of $\sim 10$ lower in 2005-08 than in 1984 when it was reported to have been detected the last time.

3) We do not see any significant change in luminosity, position or width if the broad $\mathrm{H} \alpha$ line between the two epochs it was detected in 2005-08. The average width is $4200 \mathrm{~km} \mathrm{~s}^{-1}$, consistent with the width observed in 1984. Due to the weakness of the broad emission lines we are unable to study the connection between continuum and line luminosity or between the BLR velocity field and the proposed periastron of the secondary black hole in 2005-07.

\section{References}

Adelman-McCarthy, J. K., Agüeros, M. A., Allam, S. S., et al. 2007, ApJS, 172, 634

Appenzeller, I., Fricke, K., Fürtig, W., et al. 1998, The Messenger, 94, 1

Fiorucci, M., \& Tosti, G. 1996, A\&AS, 116, 403

Katz, J. I. 1997, ApJ, 478, 527

Lehto, H. J., \& Valtonen, M. J. 1996, ApJ, 460, 207

McGill, K. L., Woo, J., Treu, T., \& Malkan, M. A. 2008, ApJ, 673, 703

Miller, J. S., French, H. B., \& Hawley, S. A. 1978, in BL Lac Objects, ed. A. M. Wolfe, 176

Sbarufatti, B., Treves, A., Falomo, R., et al. 2005, AJ, 129, 559

Schneider, D. P., Hall, P. B., Richards, G. T., et al. 2007, AJ, 134, 102

Sillanpää, A., Haarala, S., Valtonen, M. J., Sundelius, B., \& Byrd, G. G. 1988, ApJ, 325, 628

Sitko, M. L., \& Junkkarinen, V. T. 1985, PASP, 97, 1158

Stickel, M., Fried, J. W., \& Kuehr, H. 1989, A\&AS, 80, 103

Sundelius, B., Wahde, M., Lehto, H. J., \& Valtonen, M. J. 1997, ApJ, 484, 180

Valtaoja, E., Teräsranta, H., Tornikoski, M., et al. 2000, ApJ, 531, 744

Valtonen, M. J. 2007, ApJ, 659, 1074

Valtonen, M. J., Lehto, H. J., Sillanpää, A., et al. 2006, ApJ, 646, 36

Valtonen, M. J., Nilsson, K., Villforth, C., et al. 2009, ApJ, 698, 781

Villata, M., Raiteri, C. M., Sillanpaa, A., \& Takalo, L. O. 1998, MNRAS, 293, L13

Villforth, C., Nilsson, K., Heidt, J., et al. 2010, MNRAS, 402, 2087 